\section{Kidney Blood Pressure Research}

\title{
Loss of the Protein Cystathionine $\beta$-Synthase During Kidney Injury Promotes Renal Tubulointerstitial Fibrosis
}

\author{
Xiangning Yuan ${ }^{\mathrm{a}}$ Jin Zhang ${ }^{\mathrm{a}}$ Feifei Xie ${ }^{\mathrm{a}} \quad$ Wenqing Tan ${ }^{\mathrm{a}} \quad$ Shuting Wang ${ }^{\mathrm{a}}$ \\ Ling Huang $^{\mathrm{a}}$ Lijian Tao ${ }^{\mathrm{a}}$ Qiqi Xing ${ }^{\mathrm{b}}$ Qiongjing Yuan ${ }^{\mathrm{a}}$
}

aDivision of Nephrology, Department of Internal Medicine, Xiangya Hospital; ${ }^{b}$ Xiangya School of Medicine, Central South University, Changsha, Hunan, China

\section{Key Words}

Renal tubulointerstitial fibrosis $•$ Cystathionine $\beta$-synthase $・$ Renal inflammation

\begin{abstract}
Background/Aims: Renal tubulointerstitial fibrosis (TIF) is the common pathway of progressive chronic kidney disease. Inflammation has been widely accepted as the major driving force of TIF. Cystathionine $\beta$-synthase (CBS) is the first and rate-limiting enzyme in the transsulfuration pathway. CBS is considered to play protective role in liver and pulmonary fibrosis, but its role in TIF remains unknown. The purpose of this study was to investigate the potential role and mechanism of CBS in renal inflammation and TIF. Methods: Renal function, tubulointerstitium damage index score, extracellular matrix $(E C M)$ deposition, and the expressions of collagen I, collagen III, fibronectin, CD3, CD68, IL-1 $\beta$, TNF- $\alpha$ were measured in sham operation and unilateral ureteral obstruction (UUO) rats. Proteomics and gene array analysis were performed to screen differentially expressed molecules in the development of renal inflammation and TIF in UUO rats. The expression of CBS was detected in patients with obstructive nephropathy and UUO rats. We confirmed the expression of CBS using western blot and real-time PCR in HK-2 cells. Overexpression plasmid and siRNA were transfected specifically to study the possible function of CBS in HK-2 cells. Results: Abundant expression of CBS, localized in renal tubular epithelial cells, was revealed in human and rat renal tissue, which correlated negatively with the progression of fibrotic disease. Expression of CBS was dramatically decreased in the obstructed kidney from UUO rats as compared with the sham group (SHM). In addition, knocking down CBS exacerbated extracellular matrix (ECM) deposition, whereas CBS overexpression attenuated TGF- $\beta 1$-induced ECM deposition in vitro. Inflammatory and chemotactic factors were also increased in CBS knockdown HK-2 cells stimulated by IL-1 $\beta$.
\end{abstract}




\section{Kidney Blood Pressure Research}

Conclusions: These findings establish CBS as a novel inhibitor in renal fibrosis and as a new therapeutic target in patients with chronic kidney disease.

\section{Introduction}

Chronic kidney disease (CKD) is a global public health issue with increasing attributable mortality and incidence and prevalence of end-stage kidney disease (ESRD), which leads to a rising burden worldwide [1]. Renal tubulointerstitial fibrosis (TIF) is the common pathway of progressive CKD with different etiologies. The characteristic pathological manifestations of TIF are the continuous deposition of extracellular matrix (ECM), such as fibronectin (FN) and collagens, accompanied by renal tubular atrophy and renal vascular remodeling, which leads to irreversible loss of nephron construction and function in the form of ESRD [2]. Thus, preventing or postponing renal tubulointerstitial fibrosis in the early stages of CKD is a critical strategy to prevent and treat ESRD [3].

Renal tubulointerstitial inflammatory responses have multiple consequences-some harmful while others healing, is one of the four pivotal cellular responses of renal fibrosis [4]. Renal fibrosis is almost always preceded by the infiltration of inflammatory cells, including lymphocytes, monocytes/macrophages, dendritic cells, and mast cells [5]. Although inflammation is an integral part of the host defense mechanisms in response to injury, nonresolving inflammation is a major driving force in the development of fibrotic disease [59]. The presence of factors, such as interleukin-1 $\beta(\mathrm{IL}-1 \beta)$, transforming growth factor- $\beta_{1}$ (TGF- $\beta_{1}$ ) et al. $[10,11]$, while the renal inflammatory process persists, leads to development of interstitial fibrosis [12].

Renal fibrotic disorders are generally refractive to current therapies [3]. However, the concrete and fundamental mechanisms of renal inflammation remain elusive and effective drugs are scarce. Despite the importance of inflammation in renal tubulointerstitial fibrosis, the pathogenesis of renal inflammation has not been fully explained until now, it is believed to involve some novel proteins, mechanisms, or drug targets associated with the inflammation in the pathogenesis of TIF.

In the present study, we used isobaric tags for relative and absolute quantitation (ITRAQ) and gene array to analyze the differential expression of proteins and genes in the kidney of sham and unilateral ureteral obstruction (UUO) rats. Cystathionine $\beta$-synthase (CBS) was identified as one of the most significantly down-regulated proteins in the renal cortex of UUO rats. CBS, enriched in the outer cortex, especially in cells of the proximal convoluted tubule, is the first and rate-limiting enzyme in the transsulfuration pathway [13]. Homocysteine (Hcy) is detoxified through the transsulfuration pathway. It has been reported that the progression of renal inflammation and fibrosis is associated with abnormal metabolism of Hcy, a precursor of $\mathrm{H}_{2} \mathrm{~S}$ [14-16]. Recent human and animal studies have demonstrated the involvement of $\mathrm{H}_{2} \mathrm{~S}$ in various diseases, including renal inflammatory damage, TIF, and ESRD [17-19]. CBS deficiency may lead to pro-inflammatory states [20] such as vascular remodeling [21], impaired angiogenesis [22] and endothelial dysfunction [23]. However, whether CBS has certain roles in TIF is still unknown. In view of the importance of CBS in the transsulfuration pathway, the relationship among homocysteine, $\mathrm{H}_{2} \mathrm{~S}$, renal inflammation, and TIF, we hypothesize that CBS may play important role in renal inflammation and TIF.

In this study, we found that CBS is down-regulated in rat and human TIF using a systemic biology method. Moreover, the decreased CBS expression was accompanied by infiltration of inflammatory cells, expression of IL-1 $\beta$, and deposition of ECM. In vitro cell studies in which CBS was overexpressed or knocked down highlighted the fundamental role of CBS in regulating the amount of fibronectin and collagen I associated with TGF- $\beta_{1}$ stimulation. In addition, we found that silencing CBS in vitro increased the expression of inflammatory factors (TNF- $\alpha$, IL-6) and chemokines (MCP-1) in HK-2 cells stimulated by IL$1 \beta$. Taken together, to our knowledge, this presents the first investigation of the mechanistic involvement of CBS in the progression of renal inflammation and interstitial fibrosis. 


\section{Kidney Blood Pressure Research}

\begin{tabular}{l} 
Kidney Blood Press Res 2017;42:428-443 \\
\begin{tabular}{l|l}
\hline DOI: $10.1159 / 000479295$ & C 2017 The Author(s). Published by S. Karger AG, Basel \\
Published online: July 27, 2017 & www.karger.com/kbr
\end{tabular} \\
\hline
\end{tabular}

Yuan et al.: Cystathionine beta-Synthase Prevents Renal Tubulointerstitial Fibrosis

\section{Materials and Methods}

\section{Ethics statement}

This study was approved by the Ethics Committee of the Xiangya Hospital (Central South University, Hunan, China, Permit number: 201312477). Human kidney sections were obtained from surgical specimens of obstructive nephropathy (without other disease) and non-tumor normal tissue adjacent to renal carcinoma. All patients signed official informed consent forms before operation, allowing retention of their information in the hospital database for research. All animals were subject to the Care and Use of Laboratory Animals of the Ministry of Science and Technology of China guidelines. Moreover, all methods involving humans were tested in accordance with relevant guidelines and regulations.

\section{Reagents and Antibodies}

Recombinant Human Transforming Growth Factor $\beta_{1}$ (TGF- $\beta_{1}$ ) was purchased from Peprotech (USA). Recombinant Human Interleukin-1 $\beta$ (IL-1 $\beta$ ) was purchased from Cell Signaling Technology (USA) . The primary antibodies to CBS, fibronectin, collagen I and collagen III were purchased from Abcam (UK). CD68 antibody was purchased from AbD Serotec (UK), and CD3 antibody was purchased from BD Pharmingen (USA). The primary antibody to $\beta$-actin and secondary antibodies were purchased from Sigma-Aldrich (USA). The BCA Protein Assay Kit was purchased from Thermo (USA). IL-1 $\beta$ and TNF- $\alpha$ enzyme-linked immunosorbent assay (ELISA) kits were purchased from R\&D (USA). CBS, TNF- $\alpha$, MCP-1, and IL-6 quantitative polymerase chain reaction (qPCR) primer was purchased from Generay Biotech Co., Ltd (Shanghai, CHINA). CBS siRNA and control siRNA were purchased from GenePharma Co., Ltd (Suzhou, CHINA). Expression plasmids encoding human PcDNA3.1 and PcDNA3.1-CBS were obtained from Open BioSystems (Changsha, CHINA). Lipofectamine $^{\mathrm{TM}} 2000$ Transfection Reagent was purchased from Invitrogen (USA).

\section{Animals}

Male Sprague-Dawley rats (180 to 200g) were purchased from Slac Laboratory Animal Center (Shanghai, CHINA). Animals were housed in a pathogen-free environment with a 12-hour light-dark cycle and unrestricted access to a standard rat chow diet and water. Rats were randomly assigned into the sham operation group and the UUO group, with 5 rats in each group. UUO was induced by ligation of the left ureter according to the procedure previously described [24,25]. All the rats were sacrificed at 14 days after the sham or UUO operation. Half of the left kidney was harvested and fixed in 10\% neutral buffered formalin for pathologic examination and immunohistochemistry assay. The remaining half of the left kidney was preserved in liquid nitrogen for other analyses.

\section{Quantitative proteomics using iTRAQ labeling and mass spectrometry}

For ITRAQ screening, the renal cortexes isolated from SHM rats and UUO rats were lysed several times by freeze-thawing, followed by sonication. The extracted proteins were reduced and alkylated as described in the iTRAQ protocol (Applied Biosystems, Foster City, CA, USA). Isobaric tagging iTRAQ reagent (1 unit in ethanol) was added directly to the protein digest (70\% ethanol final), and the mixture was incubated. Proteins were subjected to the conventional procedure with off-line 2D LC-MS/MS. Peptides were eluted with a linear gradient. Then, the LC-MS/MS analysis was conducted with samples injected into the trap column. To identify the peptides, database searches were performed with ProteinPilot 2.0.1 software (Applied Biosystems) using Paragon and ProGroup algorithms against the Swiss-Prot rat database [26]. We selected differentially expressed proteins using peptide confidence $>95 \%$ criteria, and fold-changes $<0.7$ were set as cut-off values. The differentially expressed proteins were matched with the results of gene array through the code of SwissProt. If the protein levels reached the requirements of expression fold-changes $<0.7$ compared to SHM , we regarded it as a meaningful differential protein.

\section{Gene Array}

Total RNA was isolated from the renal cortex with Trizol Reagent. RNA was then purified with QIAGEN RNeasy mini columns (QIAGEN, USA) and re-suspended in $50 \mu$ l diethylpyrocarbonate-treated water, according to the manufacturer's protocol. Microarray analysis was conducted on the two groups by a GeneChip ${ }^{\circ}$ Rat Genome 2302.0 Array (Rat 230 2.0) (Affymetrix, USA), which includes more than 31,000 probe sets representing about 28,700 well-substantiated rat genes. Average linkage clustering analysis, 


\section{Kidney Blood Pressure Research}

Kidney Blood Press Res 2017;42:428-443

\begin{tabular}{l|l}
\hline DOI: $10.1159 / 000479295$ & (C) 2017 The Author(s). Published by S. Karger AG, Basel
\end{tabular}

Published online: July 27, 2017 www.karger.com/kbr

based on a centered Pearson correlation, was implemented in the Cluster program by Java Tree view 1.0.12@ software [27]. If the RNA levels reached the requirements of mRNA expression ratio-changes $<0.4$ compared to SHM, we regarded it as a meaningful differential gene.

\section{Histopathological assessment and immunohistochemistry}

Formalin-fixed kidneys were embedded in paraffin and prepared in $4 \mu \mathrm{m}$ sections for hematoxylineosin (HE) and Masson trichrome staining. The tubulointerstitial damage index and tubulointerstitial fibrosis score were graded as previously described [28].

Immunohistochemistry (IHC) staining was performed in paraffin sections as previously reported [29]. Slides were incubated with a primary antibody against collagen I (1:500), collagen III (1:800), CD3 (1:200), CD68 (1:200), or CBS (1:500). The positive staining cell numbers of CD3 and CD68 in 20 randomly selected fields at 400x magnifications in the cortex and outer medulla were analyzed. The staining of collagen I, collagen III, and CBS was measured in 10 randomly selected fields at 200x magnifications in the cortex using computerized morphometry (ImagePro Plus 6.0 software, Media Cybernetics, Bethesda, MD). All of the analyses were conducted by two individual pathologists in a double-blind manner as previously described [30].

\section{qPCR}

Total RNA was extracted from kidney tissue using Trizol (Life Technologies, USA) reagent according to the manufacturer's instructions. The cDNAs were synthesized from $1 \mu \mathrm{g}$ of the total RNA in a $20 \mu \mathrm{l}$ reaction system using a RevertAid First Strand cDNA Synthesis Kit (Life Technologies, USA). The specific primers used for CBS and $\beta$-actin were designed based on the GenBank sequences and synthesized by Generay Biotech Co., Ltd (Shanghai, CHINA). The CBS primer pair consisted of forward primer 5'-GACCAAGTTCCTGAGCGACA-3' and reverse primer 5'-CGGAGGATCTCGATGGTGTG-3', the collagen a (1)I primer pair consisted of forward primer 5'-CAACAATTCCTGGCGTTACCTT-3' and reverse primer 5'-AAGCCCTGTATTCCGTCTCCTT-3' \the MCP-1 primer pair consisted of forward primer 5'-CTCAGCCAGATGCAATCAAT-3' and reverse primer 5'-GCTTCTTTGGGACACTTGCT-3', the TNF- $\alpha$ primer pair consisted of forward primer 5'CCTGTGAGGAGGACGAACAT-3' and reverse primer 5'- TTTGAGCCAGAAGAGGTTGAG-3', the IL-6 primer pair consisted of forward primer 5'- CTTCGGTCCAGTTGCCTTCT-3' and reverse primer 5'- GTGAGTGGCTGTCTGTGTGG-3'. $\beta$-actin was utilized as a loading control with forward primer 5'-TCAGGTCATCACTATCGGCAAT-3' and reverse primer 5'-AAAGAAAGGGTGTAAAACGCA-3'. The qPCR quantitation for individual target mRNA expression was performed with a CFX96 Real-time Detection System (Bio-Rad, USA) using a Thermo SYBR Green qPCR kit (Thermo, USA). The amount of specific mRNA in each sample was calculated based on the cycle threshold (CT) values, which were standardized with the quantity of the housekeeping gene $\beta$-actin. Further calculation and statistical analysis was based on the comparative $2^{-\Delta \Delta C \mathrm{~T}}$ method [31].

\section{Cell culture and transfection}

Human proximal tubular epithelial cells (HK-2) were purchased from American Type Culture Collection (ATCC, Rockville, MD, U.S.). HK-2 cells were cultured in Dulbecco's Modified Eagle's Medium (DMEM, Gibco, USA) containing 10\% fetal bovine serum (FBS, Gibco, USA), $100 \mathrm{U} / \mathrm{ml}$ penicillin, and 100 g/ $\mathrm{ml}$ streptomycin (Gibco, USA) in a $5 \% \mathrm{CO}_{2}$ incubator at $37^{\circ} \mathrm{C}$.

Cells were seeded into 12-well plates in complete culture medium and then simulated with $10 \mathrm{ng} /$ $\mathrm{mL}$ of recombinant human TGF- $\beta_{1}$ for $12 \mathrm{~h}, 24 \mathrm{~h}$, and $48 \mathrm{~h}$ respectively. Whole cell protein was collected for Western blot analyses.

For transfection experiments, cells were seeded into 12-well culture plates in complete medium containing 10\% FBS without penicillin or streptomycin for $24 \mathrm{~h}$. Transfections were performed with siRNA or overexpression plasmid using Lipofetamine 2000 according to the manufacturer's instructions. The humo CBS siRNA oligos were as follows: 5'-GGCGGCUGAAGAACGAAAUTTAUUUCGUUCUUCAGCCGCCTT-3'. Six hours after transfection, cell culture mediums were exchanged for fresh medium with or without $10 \mathrm{ng} /$ $\mathrm{mL}$ of recombinant human TGF- $\beta_{1}$, and whole cell protein or RNA was collected at $48 \mathrm{~h}$ after transfection. To test the influence of CBS on inflammation, cells were incubated with $5 \mathrm{ng} / \mathrm{mL}$ of recombinant human IL-1 $\beta$ at $24 \mathrm{~h}$ after transfection with CBS siRNA, cell RNA was collected at $48 \mathrm{~h}$ after transfection. 


\section{Kidney Blood Pressure Research}

\section{Western blot analysis}

Kidney tissue and cells were lysed with a buffer containing $20 \mathrm{mM}$ Tris-HCl [pH 7.4], 4\% sodium dodecyl sulfate, and $10 \%$ glycerol. Lysates were boiled at $100^{\circ} \mathrm{C}$ for 10 minutes. Protein concentration was determined using the BCA Protein Assay Kit according to the manufacturer's protocol. Proteins were separated by $8 \%$ or $10 \%$ sodium dodecyl sulphate-polyacrylamide gel electrophoresis (SDS-PAGE) and transferred to polyvinylidenedifluoride (PVDF) membranes (Millipore, USA). The membranes were incubated in 1X TBS-T [0.05\% Tween 20, $20 \mathrm{mmol} / \mathrm{l}$ Tris- $\mathrm{HCl}$, and $150 \mathrm{mmol} / \mathrm{l} \mathrm{NaCl}$ (pH 7.6)] containing 5\% skim milk for 1 hour at room temperature before incubation overnight at $4{ }^{\circ} \mathrm{C}$ with the primary antibodies against CBS (1:2000), FN (1:800), or $\beta$-actin (1:5000). Membranes were washed three times with TBS-T and then incubated with HRP-conjugated secondary antibodies at 1:5000 dilution for 1 hour at room temperature. Signals were developed by enhanced chemiluminescence (ECL) reagents (GE Healthcare, England) and X-ray film (Kodak, USA) as previously described [32]. Bands were quantified using ImageJ as previously described [33].

ELISA

Renal cortex tissue homogenate was prepared using mechanical attrition treatment [34]. IL-1 $\beta$ and TNF- $\alpha$ protein concentrations were detected by ELISA kits according to manufacturer's instructions as previously described [35].

\section{Statistical Analysis}

Data are expressed as mean \pm SD. Statistical analysis of data was performed with SPSS 22.0 software (SPSS Inc., Il, USA). Comparison among groups was made with one-way ANOVA. Multiple-comparison tests were applied only when a significant difference was determined by the ANOVA; p-values $<0.05$ were considered to be statistically significant. Each experiment was repeated at least three times with similar results.

\section{Results}

Kidneys from UUO rats developed TIF and displayed significant inflammation

UUO is a well-established experimental model of obstructive nephropathy characterized by increased ECM deposition, which leads to tubulointerstitial fibrosis. We observed increased blood urea nitrogen (Bun), serum creatinine (Scr), and serum uric acid (UA) in UUO rats compared with those in SHM (Fig. 1A-C), which was consistent with previous study [25]. The renal tubulointerstitial injury score (Fig. 1D-E) and ECM deposition (Fig. 1F-G) were significantly increased in the obstructed kidney, confirmed by HE and Masson's trichrome staining, respectively. More specifically, immunohistochemistry staining confirmed that collagen I and collagen III deposition in the renal interstitium was markedly elevated (Fig. $1 \mathrm{H}-\mathrm{K})$, and western blot indicated enhanced fibronectin accumulation in the UUO kidney (Fig. 1L-M). In addition to ECM deposition, we analyzed the inflammation state in UUO rats. Immunohistochemistry staining showed increased infiltration of CD3-positive lymphocytes and CD68-positive macrophages in the renal interstitium after UUO operation (Fig. 2A-D). ELISA analysis detected that the protein concentration of inflammation cytokines in the renal cortex tissue homogenate, IL- $1 \beta$ and TNF- $\alpha$ for example, was elevated in the UUO (Fig. $2 \mathrm{E}-\mathrm{F}$ ). These results certified that we successfully conducted the tubulointerstitial fibrosis model, and the inflammation state involved TIF.

Fig. 1. Renal tubulointerstitial fibrosis in UUO rats. Blood urea nitrogen (Bun), B. serum creatinine (Scr), C. serum uric acid (UA) in rats, D. HE stain (x200) and corresponding E. Tubulointerstitial injury score, F. Masson's trichrome staining (x200) and corresponding G. Tubulointerstitial fibrosis score. Collagen I (H) and Collagen III (J) stained by immunohistochemistry (x200) and corresponding semi-quantitative analysis results (I) and (K). Representative Western blot of FN in kidney tissue (L) and quantitative analysis of FN protein expression $(M)$. Data expressed as means $\pm \mathrm{SD}, \mathrm{n}=5$. ${ }^{\#} \mathrm{P}<0.05$ vs. sham group. 


\section{Kidney Blood Pressure Research}

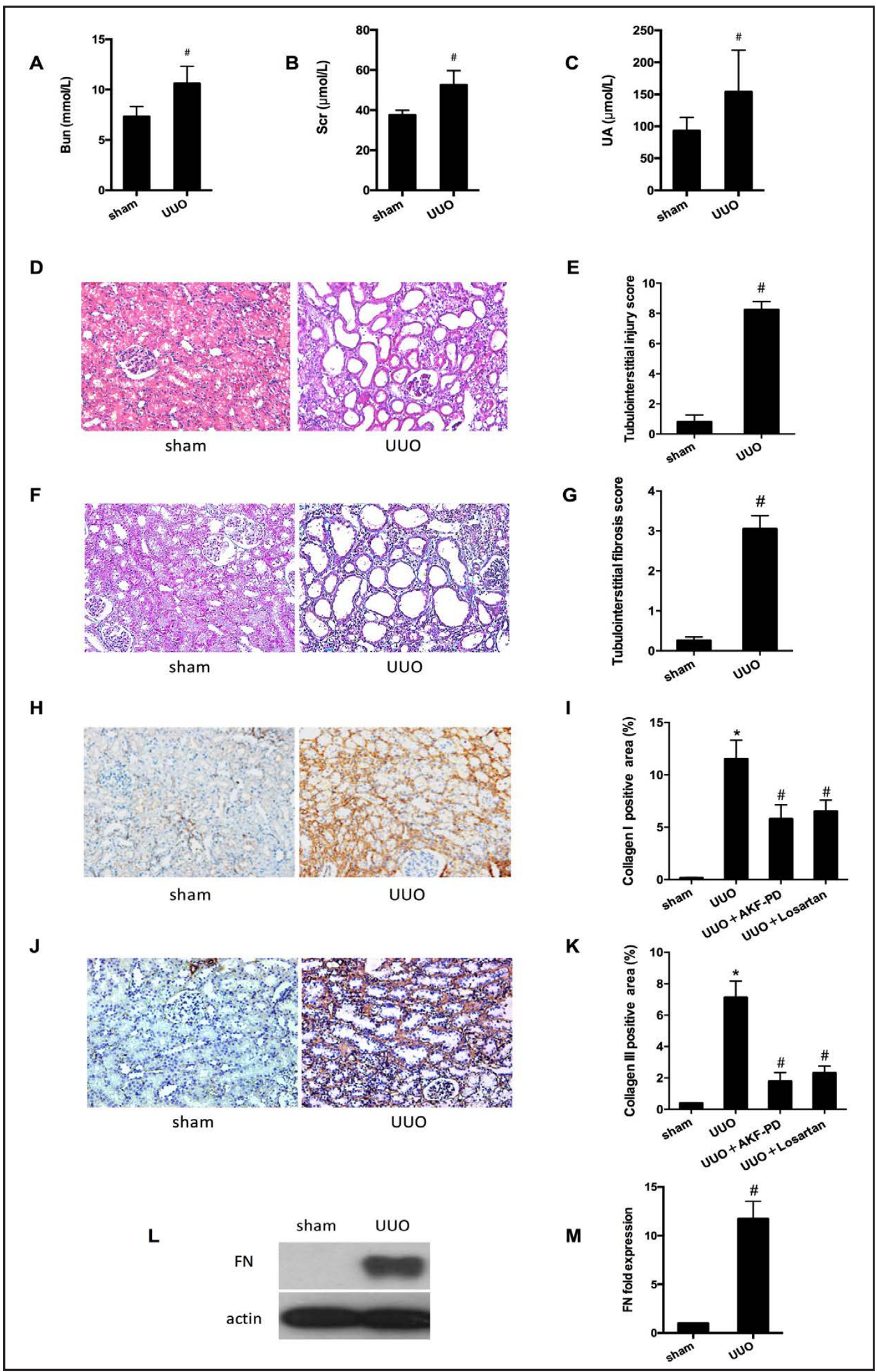




\section{Kidney Blood Pressure Research}

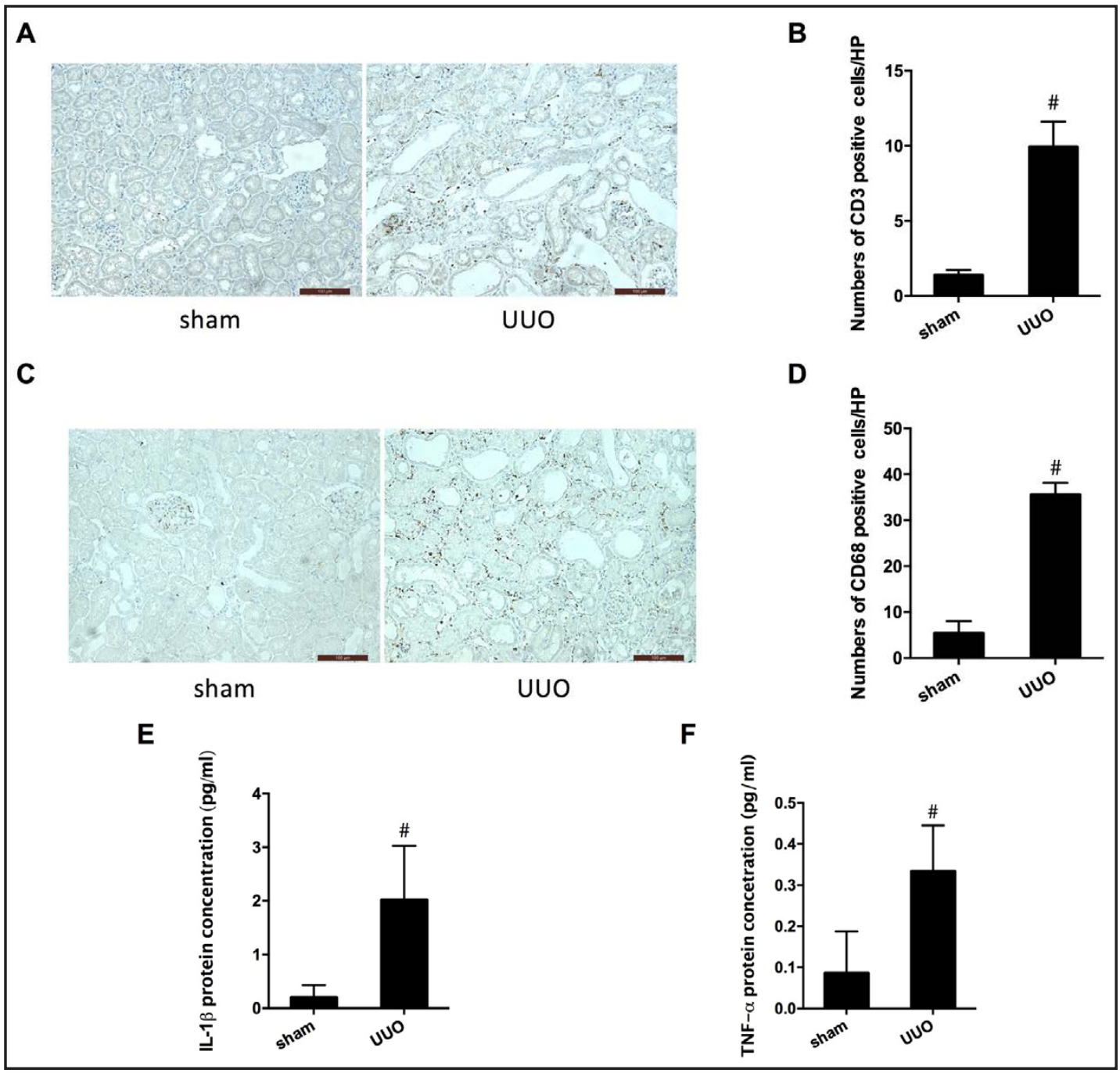

Fig. 2. Renal tubulointerstitial inflammation in UUO rats. CD68 (A) and CD3 (B) stained by immunohistochemistry (x200) and corresponding semi-quantitative analysis results (C) and (D). IL-1 $\beta$ (E) and TNF- $\alpha$ (F) protein concentrations in UUO renal tissue homogenate detected by ELISA. Data expressed as means \pm SD, $\mathrm{n}=5$. ${ }^{*} \mathrm{P}<0.05$ vs. sham group.

CBS expression was significantly decreased in the renal cortex of UUO rats

To assess the potential target proteins involved in TIF, we extracted renal cortex protein of UUO rats for proteomic and gene array analysis. Proteomic analysis yielded a total of 1105 proteins, which were identified by Protein Pilot at a confidence level of $95 \%$. Through the primary proteomic analysis in the protein data bank of Swiss-Prot, we detected 294 proteins with fold-changes $<0.7$. After matching these proteins to the differentially expressed gene detected by the United Affymetrix GeneChip profiling with ratio-changes $<0.4,18$ proteins were confirmed to be down-regulated at the transcriptional level as well as translational level in the renal cortex of UUO rats compared with those in SHM. Among them, we successfully found the CBS protein (Table 1).

To further examine CBS expression in UUO rats, we analyzed the protein and mRNA level expressions of CBS in rat kidney tissue. Firstly, we conducted IHC staining of CBS in rat kidney sections. As expected, CBS was mainly expressed in the cytoplasm of proximal renal tubular epithelial cells in normal kidneys, and was significantly decreased in obstructed kidneys (Fig. 3AB). Secondly, the down-regulation of CBS protein expression in UUO rat kidney tissue was con- 


\section{Kidney Blood Pressure Research}

firmed by Western blot analysis (Fig. 3C-D). Thirdly, qPCR tests were conducted to detect the mRNA expression change of CBS in the UUO model, which was proven to be reduced as well (Fig. 3E). Collectively, the above observations showed that the expression of CBS was decreased in TIF.

Table 1. Differentially expressed proteins in proteomics analysis

\begin{tabular}{cclcc}
\hline No. & $\begin{array}{c}\text { Gene Sy } \\
\text { mbol }\end{array}$ & Protein Name & $\begin{array}{c}\text { Swiss-prot } \\
\text { accession } \\
\text { number }\end{array}$ & $\begin{array}{c}\text { UUO/S } \\
\text { HM }\end{array}$ \\
\hline 1 & CALB1 & Calbindin & P07171 & 0.0373 \\
2 & RGN & Regucalcin & Q03336 & 0.1556 \\
3 & ST1C2 & Sulfotransferase 1C2 & Q9WUW8 & 0.1754 \\
4 & CGL & Cystathionine gamma-lyase & P18757 & 0.2089 \\
5 & S22A2 & Solute carrier family 22 member 2 & Q9R0W2 & 0.3221 \\
6 & DPYS & Dihydropyrimidinase & Q63150 & 0.3837 \\
7 & ACSF2 & Acyl-CoA synthetase family member 2, mitochondrial & Q499N5 & 0.4529 \\
8 & APOC3 & Apolipoprotein C-III & P06759 & 0.4529 \\
9 & GABT & 4-aminobutyrate aminotransferase, mitochondrial & P50554 & 0.4699 \\
10 & PYC & Pyruvate carboxylase, mitochondrial & P52873 & 0.4966 \\
11 & S1C2A & Sulfotransferase 1C2A & Q9WUW9 & 0.4966 \\
12 & SCLY & Selenocysteine lyase & Q68FT9 & 0.52 \\
13 & ARLY & Argininosuccinate lyase & P20673 & 0.5297 \\
14 & KHK & Ketohexokinase & Q02974 & 0.5598 \\
15 & GSHB & Glutathione synthetase & P46413 & 0.6194 \\
16 & CBS & Cystathionine beta-synthase & P32232 & 0.6194 \\
17 & M2GD & Dimethylglycine dehydrogenase, mitochondrial & Q63342 & 0.6855 \\
18 & PPBT & Alkaline phosphatase, tissue-nonspecific isozyme & P08289 & 0.6918 \\
\hline
\end{tabular}

CBS decreased in human obstructive nephropathy

Recent research has shown that CBS is closely related to fibrotic diseases [36]. However, the role of CBS in human TIF remains unclear. Our preceding results showed that CBS is down-regulated in the kidney of UUO rats. Thus, we analyzed CBS expression in human obstructive nephropathy, which is one of the most common forms of TIF without other confounding etiologies. CBS expression in 10 cases of non-tumor normal tissue adjacent to renal carcinoma and 9 patients with obstructive nephropathy (Table 2) was examined by IHC. In accordance with the literature, CBS was mainly detected in the cytoplasm of proximal renal tubular epithelial cells (Fig. 4A). A significant decrease of CBS was observed in patients with obstructive nephropathy (Fig. 4B).

CBS was down-regulated in TGF- $\beta_{1}$-stimulated HK-2 cells

To better explore the relationship between CBS and renal fibrosis, we established an in vitro model imitating fibrosis. Since CBS mostly expresses in the proximal convoluted tubule, HK-2, a human proximal tubule epithelial cell line from a normal adult human [37], was employed, and TGF- $\beta_{1}$ was used to induce TIF in vitro [32]. HK-2 cells were stimulated with $10 \mathrm{ng} / \mathrm{mL}$ TGF- $\beta_{1}$ for $12 \mathrm{~h}, 24 \mathrm{~h}$, or $48 \mathrm{~h}$ respectively. Western blot assay showed that CBS protein significantly decreased along with FN accumulation after treatment with TGF- $\beta$ for $48 \mathrm{~h}$ (Fig. 5A-C). Thus, it is intriguing to explore whether the overexpression of CBS would attenuate TGF- $\beta_{1}$-induced FN and collagen I accumulation in HK-2 cells.

Overexpression of CBS attenuated TGF- $\beta_{1}$-induced fibronectin and collagen I accumulation in HK-2 cells

We overexpressed CBS in HK-2 cells using plasmid transfection with PcDNA3.1 and PcDNA3.1-CBS with or without $10 \mathrm{ng} / \mathrm{mL}$ TGF- $\beta_{1}$ treatment for $48 \mathrm{~h}$. Notably, overexpression of CBS attenuated fibronectin and collagen I accumulation in HK-2 cells after TGF- $\beta_{1}$ treatment (Fig. 5D-H). These results suggested that CBS could contribute to alleviate TIF. To consolidate this hypothesis, we detected the influence of CBS knockdown in renal tubulointerstitial fibrosis in vitro.

Knockdown CBS enhanced TGF- $\beta_{1}$-induced fibronectin and collagen I accumulation in HK-2 cells

We knocked down CBS in HK-2 cells using siRNA oligo strand against CBS. Surprisingly, 


\section{Kidney \\ Blood Pressure \\ Research}

A

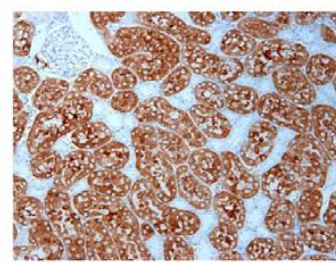

sham

C

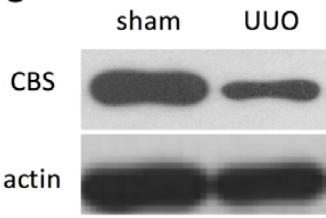

B
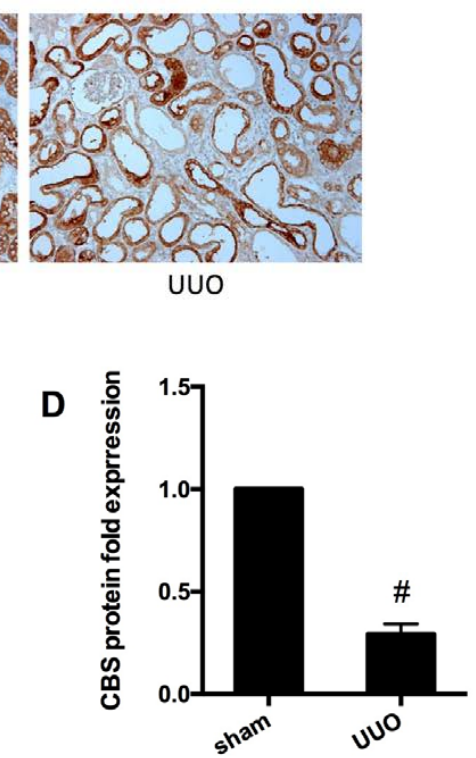

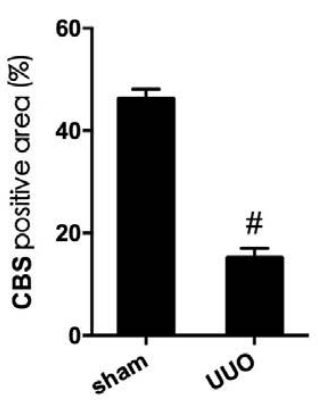

$\mathbf{E}$

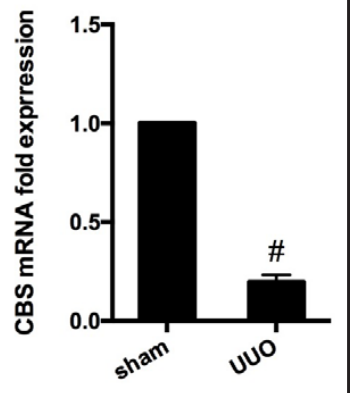

Fig. 3. Expression of CBS in UUO rats. Representative immunohistochemistry (IHC) results (200x) and corresponding B. Histogram of CBS expression in rat kidney sections. C. Representative Western blot of CBS in rat kidney tissue and D. Quantitative analysis of CBS protein expression. E. CBS mRNA expression in rat kidney tissue detected by qPCR. Data expressed as means $\pm S D, n=5$. ${ }^{*} P<0.05$ vs. sham group.

fibronectin accumulated in HK-2 cells after CBS knockdown even without the stimulation of TGF- $\beta_{1}$. Moreover, treatment with $10 \mathrm{ng} / \mathrm{mL} \quad$ TGF- $\beta$ for $48 \mathrm{~h}$ induced further accumulation of fibronectin and collagen $\mathrm{I}$ in CBS-deficient HK-2 cells (Fig. 5I-M).

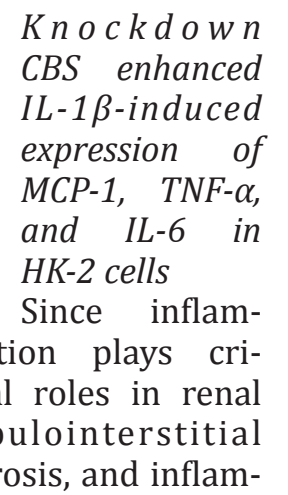

Table 2. Nephropathy patients information

\begin{tabular}{cccc}
\hline No. & Gender & $\begin{array}{c}\text { Age } \\
\text { (years) }\end{array}$ & Diagnose \\
\hline 1 & Female & 66 & Renal carcinoma (left side) \\
2 & Male & 52 & Renal carcinoma (right side) \\
3 & Female & 77 & Renal carcinoma (left side) \\
4 & Male & 53 & Renal carcinoma (right side) \\
5 & Male & 59 & Renal carcinoma (right side) \\
6 & Male & 68 & Renal carcinoma (right side) \\
7 & Female & 67 & Renal carcinoma (left side) \\
8 & Male & 64 & Renal carcinoma (right side) \\
9 & Male & 54 & Renal carcinoma (right side) \\
10 & Male & 68 & Renal carcinoma (left side) \\
11 & Female & 67 & Ureter and kidney stone, hydronephrosis (right side) \\
12 & Female & 51 & Kidney stone and hydronephrosis (right side) \\
13 & Female & 53 & Ureter and kidney stone, hydronephrosis (right side) \\
14 & Female & 53 & Kidney stone and hydronephrosis (right side) \\
15 & Male & 65 & Ureter stone and hydronephrosis (left side) \\
16 & Female & 62 & Ureter and kidney stone, hydronephrosis (left side) \\
17 & Female & 66 & Ureter and kidney stone, hydronephrosis (right side) \\
18 & Female & 67 & Ureter stone and hydronephrosis (right side) \\
19 & Male & 46 & Ureter and kidney stone, hydronephrosis (left side) \\
\hline
\end{tabular}

Control: 1-10; Obstructive Nephropathy: 11-19 


\section{Kidney Blood Pressure Research}

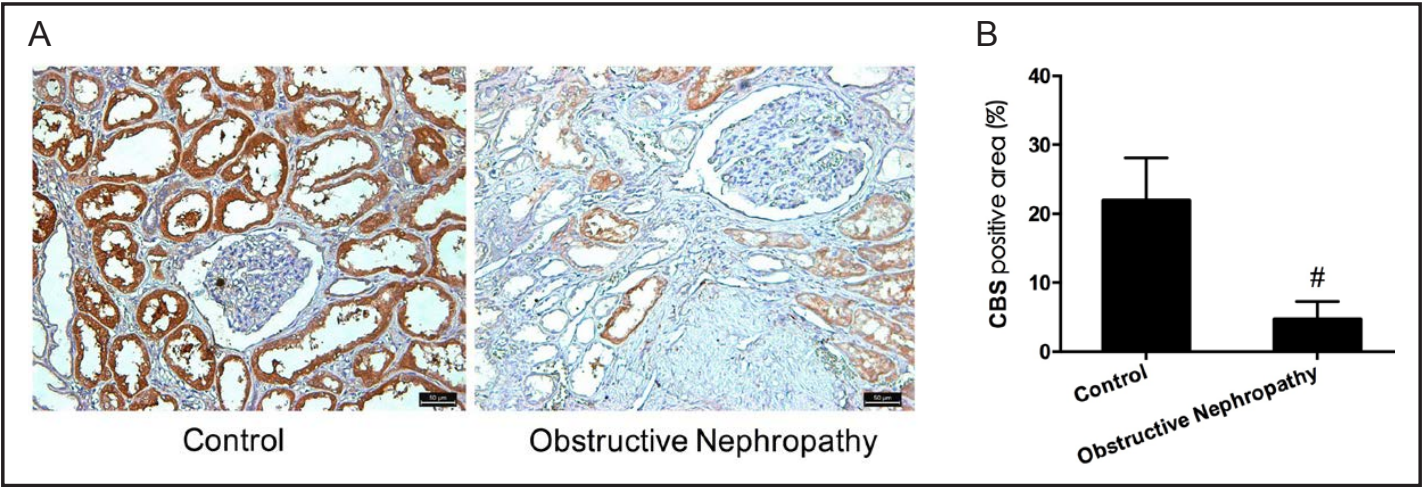

Fig. 4. Expression of CBS in human renal tissue. Representative immunohistochemistry (IHC) results (x200) and B. Histogram of control $(n=10)$ and patients with obstructive nephropathy $(n=9)$. Data expressed as means \pm SD. ${ }^{~} \mathrm{P}<0.05$ vs. Control group.

mation state is reported to be able to regulate CBS expression [38], we further explored the relationship between CBS and inflammatory reactions in TIF. We used recombined human IL-1 $\beta$ stimulation to induce inflammation in HK-2 cells. CBS mRNA was significantly decreased after treatment with $5 \mathrm{ng} / \mathrm{ml} \mathrm{IL-1} \beta$ for $24 \mathrm{~h}$ (Fig.6A). Meanwhile, marked elevation of the transcriptional expressions of MCP- 1 , TNF- $\alpha$, and IL- 6 were detected by qPCR. After transfection of siRNA against CBS, IL-1 $\beta$ induced sharply increasing mRNA expressions of MCP-1, IL-6, and TNF- $\alpha$ in the CBS deficient HK-2 cells (Fig. 6B-D).

\section{Discussion}

Understanding the fundamental pathways that lead to renal fibrosis is essential for developing better therapies for human CKD [4]. The amount of ECM deposition correlates with the severity of renal tubulointerstitial fibrosis [2]. However, our knowledge in terms of the identities of ECM accumulation-inducing factors during the pathogenesis of renal tubulointerstitial fibrosis remains limited. Although TGF- $\beta_{1}$ appears to be the primary driver of kidney fibrosis, a vast array of additional molecules may have modulating roles [4]. Additionally, TGF- $\beta_{1}$ knock-out (KO) mice develop severe multi-focal autoimmune inflammatory lesions and die within 3 weeks after birth [39], which indicates that TGF- $\beta_{1}$ may not be a good drug target, making it necessary to find additional modulating molecules. Through the proteomic and genomic analysis, we found that the introduction of inflammation and TIF was accompanied by significant reduction in CBS in the UUO model, a rodent model of fibrotic nephropathy resembling human CKD.

CBS, abundant in kidneys, livers, lungs, and other organs, is the first and rate-limiting enzyme in the transsulfuration pathway, which plays critical roles in detoxification of Hcy and production of endogenous $\mathrm{H}_{2} \mathrm{~S}[40,41]$. Hyperhomocysteinemia (HHcy) has a close relationship with several disorders in CKD [42]. Researches provide evidence to support the causal role of HHcy in the development of CKD and outlines several cellular and molecular mechanisms by which Hcy induces renal injury. These mechanisms include inflammation [43, 44], oxidative stress [45], endoplasmic reticulum stress [46], DNA hypomethylation [47,48], et al. Recent studies have revealed protective effects of $\mathrm{H}_{2} \mathrm{~S}$ in renal disease progression and failure $[16,49]$. Abnormal metabolism of $\mathrm{H}_{2} \mathrm{~S}$ is associated with fibrosis pathogenesis, causing damage to the structure and function of different organs [50]. $\mathrm{H}_{2} \mathrm{~S}$ supplementation prevents HHcy-associated renal damage, in part, through its antioxidant properties $[16,38]$.

Recently, an increasing amount of researches have indicated that CBS is closely connected with fibrotic diseases. Mani et al found that CBS-deficient mice develop inflammation, fibrosis, and hepatic steatosis [51]. Hamelet et al also observed enhanced pulmonary fibrosis 


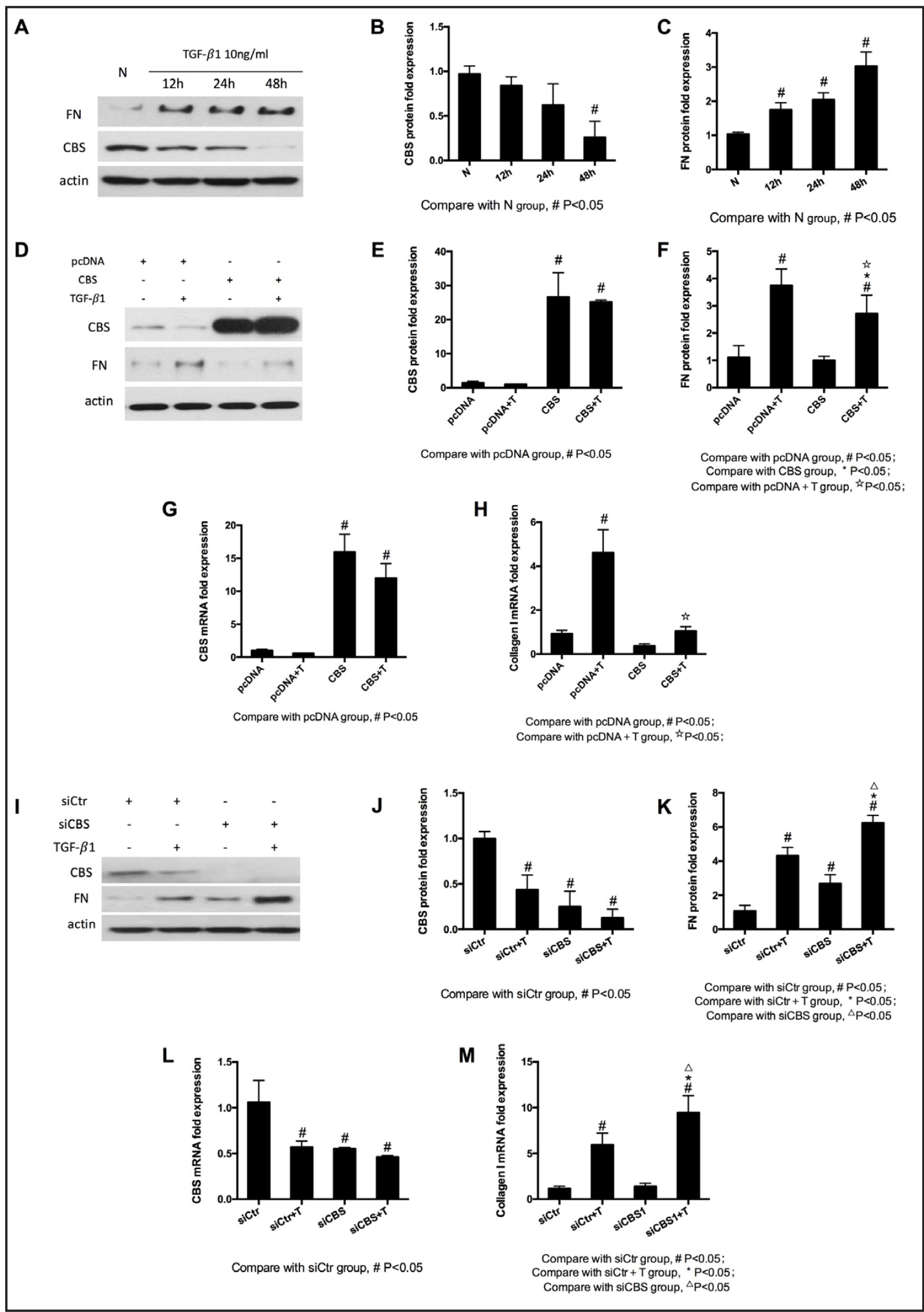

Fig. 5. CBS regulated TGF- $\beta_{1}$-induced ECM accumulation in HK-2 cells. Representative Western blot of CBS and FN in HK-2 cells treated with TGF- $\beta_{1}$ for different time, quantitative analysis of B. CBS and C. FN protein expression. D. Representative Western blot of overexpression of CBS in HK-2 cells with or without TGF- $\beta_{1}$ treatment, quantitative analysis of E. CBS and F. FN protein expression. G. CBS and H. collagen I mRNA fold 


\section{Kidney Blood Pressure Research}

expression after overexpression of CBS in HK- 2 cells with or without TGF- $\beta_{1}$ treatment, detected by qPCR. I. Representative Western blot of knockdown of CBS by siRNA in HK-2 cells with or without TGF- $\beta_{1}$ treatment, quantitative analysis of J. CBS and K. FN protein expression. L. CBS and M. collagen I mRNA fold expression after knockdown of CBS by siRNA in HK-2 cells with or without TGF- $\beta_{1}$ treatment, detected by qPCR. Data expressed as means $\pm \mathrm{SD}, \mathrm{n}=3$. . *, , , 红 $\mathrm{P}<0.05$ vs. different groups as indicated in each graph respectively.

\section{A}

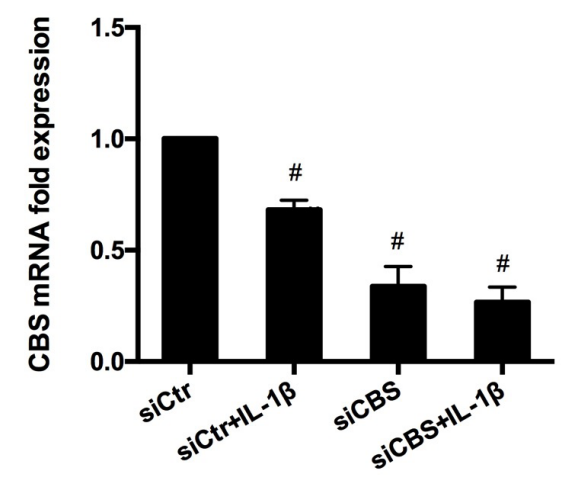

C

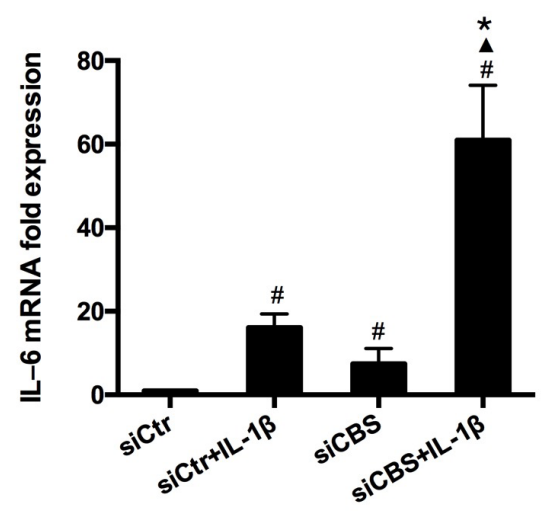

B

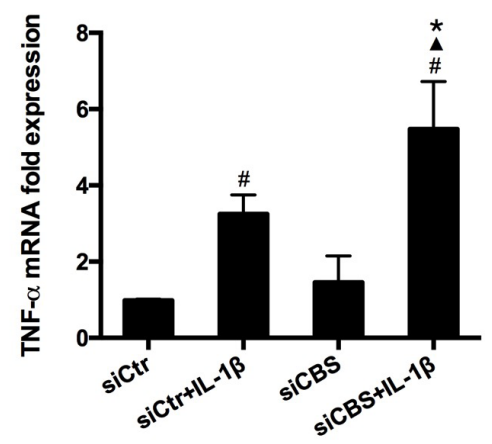

D

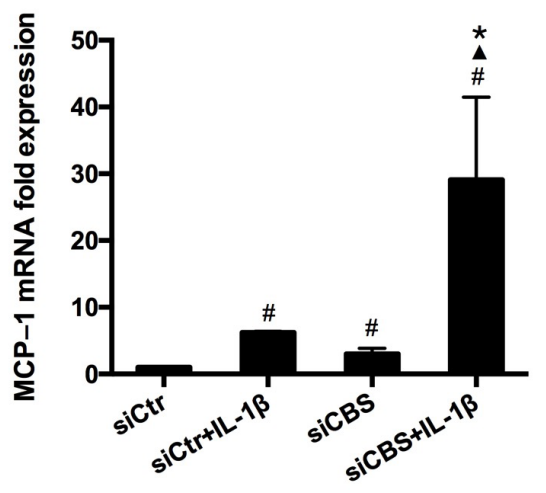

Fig. 6. CBS regulated IL-1 $\beta$-induced inflammation in HK-2 cells. A. CBS mRNA fold expression after knockdown of CBS by siRNA in HK-2 cells with or without IL-1 $\beta$ treatment. TNF- $\alpha$ (B), IL-6 (C), and MCP-1 (D) mRNA fold changes after knockdown of CBS by siRNA in HK-2 cells with or without IL-1 $\beta$ treatment. Data expressed as means $\pm \mathrm{SD}, \mathrm{n}=3$. \#,, $\triangle \mathrm{P}<0.05$ vs. different groups as indicated in each graph respectively.

in CBS-deficient mice [35]. Tan et al reported that in a carotid arterial vein patch mice model, compared with WT mice, $\mathrm{CBS}^{-/-}$mice exhibited thicker neointima with increased elastin and collagen deposition [52]. Muthuram et al conducted transverse aortic constriction (TAC) to induce pressure overload, and found that overexpression of CBS attenuated cardiac hypertrophy and fibrosis as well as reduced mortality after TAC [53]. These results suggest that CBS may be critical in protecting mammals from organ fibrosis.

Although some researches have reported the alteration of CBS expression in UUO models, almost all the studies focused on the role of $\mathrm{H}_{2} \mathrm{~S}$ instead of CBS [48,49]. The exogenous $\mathrm{H}_{2} \mathrm{~S}$, released via sodium hydrosulfide ( $\mathrm{NaHS}$ ), can ameliorate damage associated with short-term ureteral obstruction, but the reno-protective effect was depending on the direct increasing of $\mathrm{H}_{2} \mathrm{~S}$ or enhancement of CBS or both of them remained to be elucidated. Moreover, NaHS generates supra-physiological quantities of $\mathrm{H}_{2} \mathrm{~S}$ spontaneously in solution and has a half-life of only 15 minutes, which make it difficult to be a potential drug. Researcher claims that 


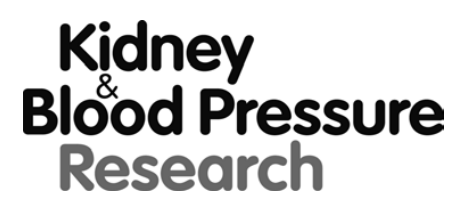

Kidney Blood Press Res 2017;42:428-443
\begin{tabular}{l|l} 
DOI: 10.1159/000479295 & (c) 2017 The Author(s). Published by S. Karger AG, Basel \\
Published online: July 27, 2017 & www.kargercom/kbr
\end{tabular}

future studies might focus on the potential to intervene fibrosis by targeting the pathway of endogenous $\mathrm{H}_{2} \mathrm{~S}$-producing enzymes [44]. However, the relationship between CBS and TIF remained to be determined. This study intended to elucidate the role and mechanism of CBS in renal interstitial inflammation and TIF.

First of all, we confirmed that the mRNA and protein expression of CBS was dramatically down-regulated in obstructive kidneys accompanied by CD3-and CD68-positive inflammation cells infiltration, IL-1 $\beta$ production, and ECM deposition. Therefore, based on the literature and our results, we postulated that CBS might be a critical regulator in inflammation and ECM deposition in kidney diseases.

Furthermore, we detected CBS expression change in obstructive nephropathy in human. Our results showed that CBS was abundantly present in normal human kidney tissue, in which the majority of renal tubules stained positive for CBS, which aligns with a previous study indicating that CBS activity was found in the renal proximal tubule [54]. As expected, to our knowledge, it is the first report that the expression of CBS was sharply down-regulated in interstitial fibrosis accompanied with ECM deposition in the kidney tissue from obstructive nephropathy patients.

Next, to further confirm the role of CBS in fibrosis, we knocked down and overexpressed CBS in TGF- $\beta_{1}$, an essential fibrogenic factor, stimulated HK- 2 cells. Results showed that knocking down CBS exacerbated ECM deposition, whereas CBS overexpression attenuated TGF- $\beta_{1}$ induced fibronectin and collagen I production. These in vitro findings are consistent with the induction of TIF accompanied by decreased CBS expression in vivo by the UUO operation. Thus, we speculated that CBS might prevent the progress of TIF.

Unresolved inflammation is one of the most important initiators that promote progressive TIF, which usually culminate in ESRD [6]. Kidney inflammation involves cells of the immune system as well as activation of intrinsic renal cells, with the consequent production and release of pro-fibrotic cytokines and growth factors that drive the fibrotic process [55]. In view of the fact that CBS deficiency may cause a pro-inflammation state [56], we speculated that the anti-fibrotic effect of CBS may partially depend on its antiinflammatory effect. As one of the crucial inflammatory cytokines, IL-1 $\beta$ initiates the production of cytokines and chemokines, exacerbates intrinsic renal cell damage [57], and mediates the disruption of interstitial ECM balance [58]. Furthermore, our results indicated that the IL-1 $\beta$ production was significantly up-regulated in UUO rats. Therefore, we chose IL-1 $\beta$ as the stimulator to induce inflammation in HK-2 cells. We found that IL-1 $\beta$ decreased CBS mRNA expression in HK-2 cells, as well as inducing a sharp increase of MCP-1, IL-6, and TNF- $\alpha$ mRNA expression in the CBS-deficient HK- 2 cells. Taken together, these findings suggested significant interrelationships between CBS expression and inflammation in renal disease.

\section{Conclusion}

In summary, this study indicated that CBS is one of the ECM accumulation-decreasing factors during the pathogenesis of renal tubulointerstitial fibrosis. The regulating effect of CBS may be mediated by its anti-inflammatory function, and overexpression of CBS may have renal protective effects. However, the concrete mechanism through which CBS exerts its anti-fibrotic and anti-inflammatory effects requires further study. What is more, our proteomics and genomic analysis showed that the hypoexpression is much more pronouced for Cystathionine gamma-lyase (CSE),another important enzyme in the transsulfuration pathway of Hcy metabolism [38], than for CBS, suggesting that the pathologic change detected in UUO rats may be also dependent on this selective impairment of CSE and that the inflammatory upraise may be relevant to a uneven handling of homocysteine and related sulfur metabolited [44]. Also other down-regulated proteins involved in calciummodulation, such as Calbindin or Regucalcin, could be interesting. Further researches are needed to explore the other down-regulated proteins roles in TIF. 


\section{Kidney Blood Pressure Research}

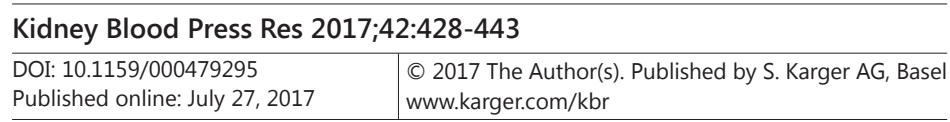

Yuan et al.: Cystathionine beta-Synthase Prevents Renal Tubulointerstitial Fibrosis

\section{Disclosure Statement}

The authors have no conflict of interest to disclose.

\section{Acknowledgment}

This project was supported by the National Natural Science Foundation of China (grants No. 81300610, No.81273575 and No. 81600582) and College students' innovative undertaking plan of Central south university(grants No.CX2015529 and CX2015530).

\section{References}

1 Fraser SD, Blakeman T: Chronic kidney disease: Identification and management in primary care. Pragmat Obs Res 2016;7:21-32.

2 Meng XM, Nikolic-Paterson DJ, Lan HY: TGF-beta: The master regulator of fibrosis. Nat Rev Nephrol 2016;12:325-338.

3 Samarakoon R, Rehfuss A, Khakoo NS, Falke LL, Dobberfuhl AD, Helo S, Overstreet JM, Goldschmeding R, Higgins PJ: Loss of expression of protein phosphatase magnesium-dependent 1A during kidney injury promotes fibrotic maladaptive repair. FASEB J 2016;30:3308-3320.

4 Eddy AA: Overview of the cellular and molecular basis of kidney fibrosis. Kidney Int Suppl (2011) 2014;4:2-8.

-5 Sun N, Zhai L, Li H, Shi LH, Yao Z, Zhang B: Angiotensin-Converting enzyme inhibitor (ACEI)-Mediated amelioration in renal fibrosis involves suppression of mast cell degranulation. Kidney Blood Press Res 2016;41:108-118.

6 Liu Y: Cellular and molecular mechanisms of renal fibrosis. Nat Rev Nephrol 2011;7:684-696.

7 Schroder K, Tschopp J: The inflammasomes. Cell 2010;140:821-832.

8 Nathan C, Ding A: Nonresolving inflammation. Cell 2010;140:871-882.

-9 Ke Y, Tang H, Ye C, Lei CT, Wang YM, Su H, Zhang C, Jiang HJ: Role and association of inflammatory and apoptotic caspases in renal tubulointerstitial fibrosis. Kidney Blood Press Res 2016;41:643-653.

10 Zuccala' A, Cianci R, Presta P, Fuiano G: [Nephroangiosclerosis and ischemic nephropathy: Two different entities or two renal manifestations of the same systemic cardiovascular disease?]. G Ital Nefrol 2009;26:299-309.

11 Chang YK, Choi H, Jeong JY, Na KR, Lee KW, Choi DE: Co-inhibition of angiotensin II receptor and endothelin-1 attenuates renal injury in unilateral ureteral obstructed mice. Kidney Blood Press Res 2016;41:450-459.

12 Bohle A, Muller GA, Wehrmann M, Mackensen-Haen S, Xiao JC: Pathogenesis of chronic renal failure in the primary glomerulopathies, renal vasculopathies, and chronic interstitial nephritides. Kidney Int Suppl 1996;54:S2-S9.

13 Kraus JP, Janosik M, Kozich V, Mandell R, Shih V, Sperandeo MP, Sebastio G, de Franchis R, Andria G, Kluijtmans LA, Blom H, Boers GH, Gordon RB, Kamoun P, Tsai MY, Kruger WD, Koch HG, Ohura T, Gaustadnes M: Cystathionine beta-synthase mutations in homocystinuria. Hum Mutat 1999;13:362-375.

14 Aminzadeh MA, Vaziri ND: Downregulation of the renal and hepatic hydrogen sulfide (H2S)-producing enzymes and capacity in chronic kidney disease. Nephrol Dial Transplant 2012;27:498-504.

15 Perna AF, Luciano MG, Ingrosso D, Pulzella P, Sepe I, Lanza D, Violetti E, Capasso R, Lombardi C, De Santo NG: Hydrogen sulphide-generating pathways in haemodialysis patients: A study on relevant metabolites and transcriptional regulation of genes encoding for key enzymes. Nephrol Dial Transplant 2009;24:37563763.

16 Sen U, Basu P, Abe OA, Givvimani S, Tyagi N, Metreveli N, Shah KS, Passmore JC, Tyagi SC: Hydrogen sulfide ameliorates hyperhomocysteinemia-associated chronic renal failure. Am J Physiol Renal Physiol 2009;297:F410-F419.

17 Lin S, Visram F, Liu W, Haig A, Jiang J, Mok A, Lian D, Wood ME, Torregrossa R, Whiteman M, Lobb I, Sener A: GYY4137, a Slow-Releasing hydrogen sulfide donor, ameliorates renal damage associated with chronic obstructive uropathy. J Urol 2016;196:1778-1787. 


\section{Kidney \\ Blood Pressure Research}

18 Kimura H: Hydrogen sulfide: Its production, release and functions. Amino Acids 2011;41:113-121.

19 Zhou X, Feng Y, Zhan Z, Chen J: Hydrogen sulfide alleviates diabetic nephropathy in a streptozotocininduced diabetic rat model. J Biol Chem 2014;289:28827-28834.

20 Kamath AF, Chauhan AK, Kisucka J, Dole VS, Loscalzo J, Handy DE, Wagner DD: Elevated levels of homocysteine compromise blood-brain barrier integrity in mice. Blood 2006;107:591-593.

-21 Tyagi N, Qipshidze N, Sen U, Rodriguez W, Ovechkin A, Tyagi SC: Cystathionine beta synthase gene dose dependent vascular remodeling in murine model of hyperhomocysteinemia. Int J Physiol Pathophysiol Pharmacol 2011;3:210-222.

22 Bosch-Marce M, Pola R, Wecker AB, Silver M, Weber A, Luedemann C, Curry C, Murayama T, Kearney M, Yoon YS, Malinow MR, Asahara T, Isner JM, Losordo DW: Hyperhomocyst(e)inemia impairs angiogenesis in a murine model of limb ischemia. Vasc Med 2005;10:15-22.

23 Saha S, Chakraborty PK, Xiong X, Dwivedi SK, Mustafi SB, Leigh NR, Ramchandran R, Mukherjee P, Bhattacharya R: Cystathionine beta-synthase regulates endothelial function via protein S-sulfhydration. FASEB J 2016;30:441-456.

-24 Yuan Q, Wang R, Peng Y, Fu X, Wang W, Wang L, Zhang F, Peng Z, Ning W, Hu G, Wang Z, Tao L: Fluorofenidone attenuates tubulointerstitial fibrosis by inhibiting TGF-beta(1)-induced fibroblast activation. Am J Nephrol 2011;34:181-194.

25 Qin J, Mei WJ, Xie YY, Huang L, Yuan QJ, Hu GY, Tao LJ, Peng ZZ: Fluorofenidone attenuates oxidative stress and renal fibrosis in obstructive nephropathy via blocking NOX2 (gp91phox) expression and inhibiting ERK/MAPK signaling pathway. Kidney Blood Press Res 2015;40:89-99.

26 Unwin RD, Griffiths JR, Whetton AD: Simultaneous analysis of relative protein expression levels across multiple samples using iTRAQ isobaric tags with 2D nano LC-MS/MS. Nat Protoc 2010;5:1574-1582.

27 Liu J, Qin J, Mei W, Zhang H, Yuan Q, Peng Z, Luo R, Yuan X, Huang L, Tao L: Expression of Niban in renal interstitial fibrosis. Nephrology (Carlton) 2014;19:479-489.

-28 Zhang J, Zheng L, Yuan X, Liu C, Yuan Q, Xie F, Qiu S, Peng Z, Tang Y, Meng J, Qin J, Hu G, Tao L: Mefunidone ameliorates renal inflammation and tubulointerstitial fibrosis via suppression of IKKbeta phosphorylation. Int J Biochem Cell Biol 2016;80:109-118.

29 Liu C, Mei W, Tang J, Yuan Q Huang L, Lu M, Wu L, Peng Z, Meng J, Yang H, Shen H, Lv B, Hu G, Tao L: Mefunidone attenuates tubulointerstitial fibrosis in a rat model of unilateral ureteral obstruction. Plos One 2015;10:e129283.

30 Mei W, Peng Z, Lu M, Liu C, Deng Z, Xiao Y, Liu J, He Y, Yuan Q Yuan X, Tang D, Yang H, Tao L: Peroxiredoxin 1 inhibits the oxidative stress induced apoptosis in renal tubulointerstitial fibrosis. Nephrology (Carlton) 2015;20:832-842.

31 Song C, He L, Zhang J, Ma H, Yuan X, Hu G, Tao L, Zhang J, Meng J: Fluorofenidone attenuates pulmonary inflammation and fibrosis via inhibiting the activation of NALP3 inflammasome and IL-1beta/IL-1R1/ MyD88/NF-kappaB pathway. J Cell Mol Med 2016;20:2064-2077.

32 Zhang Y, Berka V, Song A, Sun K, Wang W, Zhang W, Ning C, Li C, Zhang Q, Bogdanov M, Alexander DC, Milburn MV, Ahmed MH, Lin H, Idowu M, Zhang J, Kato GJ, Abdulmalik OY, Zhang W, Dowhan W, Kellems RE, Zhang P, Jin J, Safo M, Tsai AL, Juneja HS, Xia Y: Elevated sphingosine-1-phosphate promotes sickling and sickle cell disease progression. J Clin Invest 2014;124:2750-2761.

-33 Yuan Q, Wang L, Zhang F, Wang R, Fu X, Peng Z, Ning W, Hu G, Wang Z, Tao L: Fluorofenidone suppresses epithelial-mesenchymal transition and the expression of connective tissue growth factor via inhibiting TGF-beta/Smads signaling in human proximal tubular epithelial cells. Pharmazie 2011;66:961-967.

34 Tang Y, Zhang F, Huang L, Yuan Q, Qin J, Li B, Wang N, Xie Y, Wang L, Wang W, Kwan K, Peng Z, Hu G, Li J, Tao L: The protective mechanism of fluorofenidone in renal interstitial inflammation and fibrosis. Am J Med Sci 2015;350:195-203.

35 Huang L, Zhang F, Tang Y, Qin J, Peng Y, Wu L, Wang F, Yuan Q, Peng Z, Liu J, Meng J, Tao L: Fluorofenidone attenuates inflammation by inhibiting the NF-small ka, CyrillicB pathway. Am J Med Sci 2014;348:75-80.

-36 Hamelet J, Maurin N, Fulchiron R, Delabar JM, Janel N: Mice lacking cystathionine beta synthase have lung fibrosis and air space enlargement. Exp Mol Pathol 2007;83:249-253.

-37 Ryan MJ, Johnson G, Kirk J, Fuerstenberg SM, Zager RA, Torok-Storb B: HK-2: An immortalized proximal tubule epithelial cell line from normal adult human kidney. Kidney Int 1994;45:48-57.

38 Perna AF, Zacchia M, Trepiccione F, Ingrosso D: The sulfur metabolite lanthionine: Evidence for a role as a novel uremic toxin. Toxins (Basel) 2017;9 


\section{Kidney \\ Blood Pressure Research}

-39 Bommireddy R, Bueno OF, Martin J, Ormsby I, Chen H, Gard C, Molkentin JD, Boivin GP, Babcock GF, Doetschman T: Calcineurin deficiency decreases inflammatory lesions in transforming growth factor beta1-deficient mice. Clin Exp Immunol 2009;158:317-324.

40 Beard RJ, Bearden SE: Vascular complications of cystathionine beta-synthase deficiency: Future directions for homocysteine-to-hydrogen sulfide research. Am J Physiol Heart Circ Physiol 2011;300:H13-H26.

-41 Singh S, Padovani D, Leslie RA, Chiku T, Banerjee R: Relative contributions of cystathionine beta-synthase and gamma-cystathionase to $\mathrm{H} 2 \mathrm{~S}$ biogenesis via alternative trans-sulfuration reactions. J Biol Chem 2009;284:22457-22466.

42 Suliman ME, Stenvinkel P, Qureshi AR, Barany P, Heimburger O, Anderstam B, Alvestrand A, Lindholm B: Hyperhomocysteinemia in relation to plasma free amino acids, biomarkers of inflammation and mortality in patients with chronic kidney disease starting dialysis therapy. Am J Kidney Dis 2004;44:455-465.

43 Shastry S, James LR: Homocysteine-induced macrophage inflammatory protein-2 production by glomerular mesangial cells is mediated by PI3 Kinase and p38 MAPK. J Inflamm (Lond) 2009;6:27.

\$4 Capasso R, Sambri I, Cimmino A, Salemme S, Lombardi C, Acanfora F, Satta E, Puppione DL, Perna AF, Ingrosso D: Homocysteinylated albumin promotes increased monocyte-endothelial cell adhesion and upregulation of MCP1, Hsp60 and ADAM17. Plos One 2012;7:e31388.

45 Shastry S, Ingram AJ, Scholey JW, James LR: Homocysteine induces mesangial cell apoptosis via activation of p38-mitogen-activated protein kinase. Kidney Int 2007;71:304-311.

46 Cai Y, Zhang C, Nawa T, Aso T, Tanaka M, Oshiro S, Ichijo H, Kitajima S: Homocysteine-responsive ATF3 gene expression in human vascular endothelial cells: Activation of c-Jun NH(2)-terminal kinase and promoter response element. Blood 2000;96:2140-2148.

47 Jamaluddin MD, Chen I, Yang F, Jiang X, Jan M, Liu X, Schafer AI, Durante W, Yang X, Wang H: Homocysteine inhibits endothelial cell growth via DNA hypomethylation of the cyclin a gene. Blood 2007;110:3648-3655.

-48 Ingrosso D, Cimmino A, Perna AF, Masella L, De Santo NG, De Bonis ML, Vacca M, D'Esposito M, D'Urso M, Galletti P, Zappia V: Folate treatment and unbalanced methylation and changes of allelic expression induced by hyperhomocysteinaemia in patients with uraemia. Lancet 2003;361:1693-1699.

49 Sen U, Pushpakumar SB, Amin MA, Tyagi SC: Homocysteine in renovascular complications: Hydrogen sulfide is a modulator and plausible anaerobic ATP generator. Nitric Oxide 2014;41:27-37.

50 Zhang S, Pan C, Zhou F, Yuan Z, Wang H, Cui W, Zhang G: Hydrogen sulfide as a potential therapeutic target in fibrosis. Oxid Med Cell Longev 2015;2015:593407.

-51 Mani S, Cao W, Wu L, Wang R: Hydrogen sulfide and the liver. Nitric Oxide 2014;41:62-71.

-52 Tan H, Shi C, Jiang X, Lavelle M, Yu C, Yang X, Wang H: Hyperhomocysteinemia promotes vascular remodeling in vein graph in mice. Front Biosci (Landmark Ed) 2014;19:958-966.

53 Muthuramu I, Singh N, Amin R, Nefyodova E, Debasse M, Van Horenbeeck I, Jacobs F, De Geest B: Selective homocysteine-lowering gene transfer attenuates pressure overload-induced cardiomyopathy via reduced oxidative stress. J Mol Med (Berl) 2015;93:609-618.

54 House JD, Brosnan ME, Brosnan JT: Characterization of homocysteine metabolism in the rat kidney. Biochem J 1997;328 ( Pt 1):287-292.

55 Meng XM, Nikolic-Paterson DJ, Lan HY: Inflammatory processes in renal fibrosis. Nat Rev Nephrol 2014;10:493-503.

-56 Robert K, Nehme J, Bourdon E, Pivert G, Friguet B, Delcayre C, Delabar JM, Janel N: Cystathionine beta synthase deficiency promotes oxidative stress, fibrosis, and steatosis in mice liver. Gastroenterology 2005;128:1405-1415.

57 Faubel S, Lewis EC, Reznikov L, Ljubanovic D, Hoke TS, Somerset H, Oh DJ, Lu L, Klein CL, Dinarello CA, Edelstein CL: Cisplatin-induced acute renal failure is associated with an increase in the cytokines interleukin (IL)-1beta, IL-18, IL-6, and neutrophil infiltration in the kidney. J Pharmacol Exp Ther 2007;322:8-15.

58 Osman B, Akool E, Doller A, Muller R, Pfeilschifter J, Eberhardt W: Differential modulation of the cytokineinduced MMP-9/TIMP-1 protease-antiprotease system by the mTOR inhibitor rapamycin. Biochem Pharmacol 2011;81:134-143. 